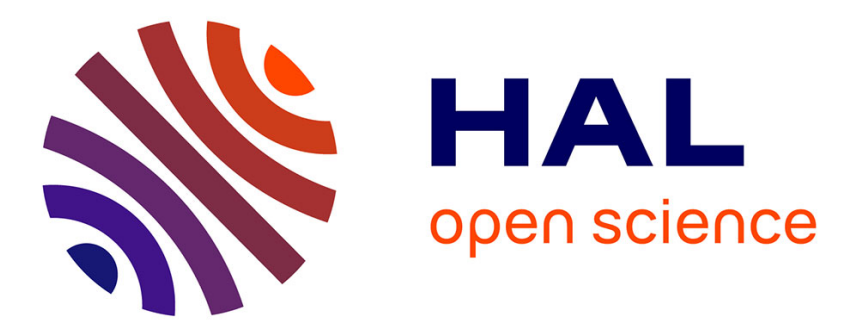

\title{
Expériences préliminaires à la réalisation d'un interrupteur semi-rapide (ms) pour courants intenses (MA)
}

B. Geoffrion

\section{- To cite this version:}

B. Geoffrion. Expériences préliminaires à la réalisation d'un interrupteur semi-rapide (ms) pour courants intenses (MA). Revue de Physique Appliquée, 1976, 11 (1), pp.181-185. 10.1051/rphysap:01976001101018100 . jpa-00243963

\section{HAL Id: jpa-00243963 https://hal.science/jpa-00243963}

Submitted on 1 Jan 1976

HAL is a multi-disciplinary open access archive for the deposit and dissemination of scientific research documents, whether they are published or not. The documents may come from teaching and research institutions in France or abroad, or from public or private research centers.
L'archive ouverte pluridisciplinaire $\mathbf{H A L}$, est destinée au dépôt et à la diffusion de documents scientifiques de niveau recherche, publiés ou non, émanant des établissements d'enseignement et de recherche français ou étrangers, des laboratoires publics ou privés. 
Classification

Physics Abstracts

0.665

\title{
EXPÉRIENCES PRÉLIMINAIRES \\ A LA RÉALISATION D'UN INTERRUPTEUR SEMI-RAPIDE (ms) POUR COURANTS INTENSES (MA)
}

\author{
B. GEOFFRION $(*)$ \\ Laboratoire de Physique des Plasmas, Groupe Fusion \\ Université Paris-Sud 91405 Orsay, France
}

(Reçu le 15 juillet 1975, révisé le 8 septembre 1975, accepté le 22 septembre 1975)

\begin{abstract}
Résumé. - Nous étudions un fusible pour courant intense (MA) à temps de montée long (s). Les essais effectués montrent qu'il est possible avec un montage simple d'atteindre des temps d'ouverture inférieurs à la milliseconde pour des coefficients de surtension de l'ordre de 500 .
\end{abstract}

Abstract. - A fuse for large currents in the megampere range with rise time of a few seconds is studied. The opening time is less than one millisecond and the overvoltage coefficient is about 500 .

1. Introduction. - Les expériences de physique sur les plasmas denses nécessitent des sources d'énergie de grande puissance. L'élément de base d'une telle source peut être une dynamo homopolaire impulsionnelle, sans fer, de grande puissance massique semblable à celle précédemment décrite [1] dont l'énergie cinétique maximale accumulée dans le rotor atteint $5 \mathrm{MJ}$.

Cette génératrice peut délivrer, selon la charge, un courant de l'ordre du mégaampère pendant un temps voisin d'une seconde. Dans le but d'obtenir des impulsions plus courtes (de quelques microsecondes) nous avons construit une bobine d'induction aux caractéristiques adaptées à celles de la machine permettant de stocker l'énergie, délivrée par la dynamo, sous forme magnétique [2]. Cette bobine a été étudiée pour atteindre le meilleur rendement possible dans le cas du transfert de l'énergie magnétique stockée vers une autre inductance $B_{2}$ couplée inductivement à la charge $B_{1}$ (Fig. 1). Le transfert aura lieu lors de l'ouverture rapide

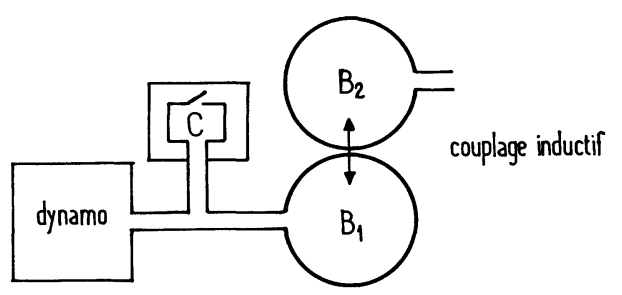

FIG. 1. - Schéma d'un transfert inductif.

$\left(^{*}\right)$ Présente adresse : Laboratoire de Génie Electrique de Paris (L. G. E. P.-L. C. I. E.) Equipe « Matière Condensée et Conversion d'Energie », 33, av. du Gal-Leclerc 92260 Fontenayaux-Roses. d'un interrupteur C placé sur le circuit d'alimentation de la bobine.

Nous décrivons ci-dessous, un interrupteur à ouverture avec lequel nous coupons un courant de 0,5 MA en un temps inférieur à la milliseconde.

Une étude comparable a été effectuée [3] sur des feuilles de cuivre explosées, mais, le temps de montée de l'impulsion de courant, produite par un générateur à compression de champ magnétique, est court (quelques dizaines de microsecondes) aussi ferons-nous une comparaison des deux types d'interrupteurs.

Nous donnons ensuite quelques résultats de calculs qui seront développés par ailleurs $\left(^{1}\right)$.

2. Dispositif expérimental. - 2.1 SOURCE DE COURANT ET MESURES. - Le dispositif expérimental est celui décrit dans un précédent article [2], il est schématisé sur la figure 2 .

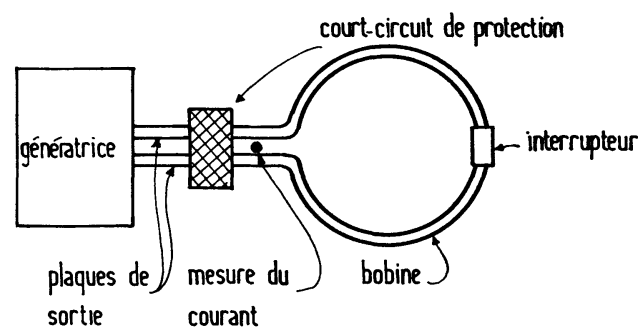

FIG. 2. - Schéma du dispositif expérimental.

La dynamo $5 \mathrm{MJ}$ est couplée à la bobine monospire qui est constituée de deux demi-cylindres permettant

(1) Allary, J. P. Communication privée. 
l'insertion de l'interrupteur. CC est un court-circuit qui protège la machine des surtensions apparaissant lors de l'ouverture brusque de l'interrupteur. En fonctionnement normal l'impédance du court-circuit est très grande avant celle de la bobine $(1 \mu \Omega, \simeq 1 \mu \mathrm{H})$.

La mesure du courant débité par la génératrice est faite à l'aide d'une sonde de Rogowsky [1] et d'un oscillographe cathodique, en raison de la rapidité du phénomène. Puisque nous enregistrons la dérivée du courant nous avons deux parties dans le signal : la première correspond à l'impulsion de courant fournie par la dynamo, la variation est lente et l'amplitude faible $(1 \mathrm{~s}, 0,5 \mathrm{~V})$ la deuxième partie correspond à la coupure rapide du courant et une forte amplitude $(1 \mathrm{~ms}$, $500 \mathrm{~V}$ ) (Fig. 3).

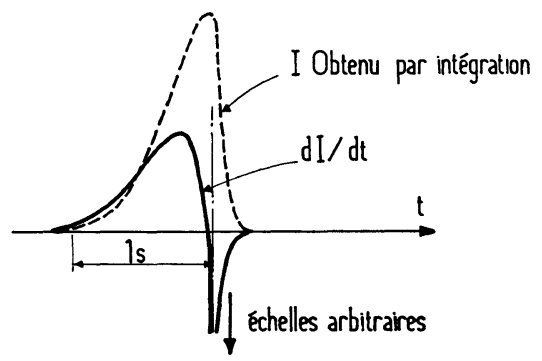

Fig. 3. - Dérivée en fonction du temps du courant dans la bobine (mesurée) et le courant (calculé).

La tension aux bornes de l'interrupteur est mesurée à l'aide de deux sondes haute tension, en montage différentiel car l'ensemble machine-bobine est isolé.

2.2 Détermination DES INTERRUPTEURS. - Les interrupteurs essayés sont du type "fusibles ». Leur section droite est déterminée de sorte que la température de fusion soit atteinte au moment du maximum de courant qui est fixé à $0,5 \mathrm{MA}$. La vitesse maximale de la dynamo doit être de 7,600 t. $\mathrm{min}^{-1}$ légèrement supérieure $\left(600 \mathrm{t} \cdot \mathrm{min}^{-1}\right)$ à la vitesse nécessaire pour obtenir le même courant dans le cas où l'interrupteur est remplacé par une pièce massive [2] ceci en raison de la légère variation de la résistance aux bornes de la génératrice [1].

Dans une première approche nous considérons la répartition du courant comme homogène, et calculons, la section droite d'un fusible de cuivre, en négligeant, la diffusion thermique, mais en tenant compte de la variation de la résistivité en fonction de la température.

En répartition homogène, le courant est de la forme $I(t)=I_{\mathrm{M}} / \mathrm{ch} a I_{\mathrm{M}} t$ [2] et un fusible dont la section droite est de $8 \mathrm{~cm}^{2}$ répond aux conditions que nous nous sommes fixés.

L'expérience est faite avec des plaques de cuivre placées aux bornes de la bobine grâce à un dispositif mécanique assurant au mieux les contacts électriques.

Les premières plaques sont rainurées comme l'indique la figure 4 (type I). Cette forme minimise la

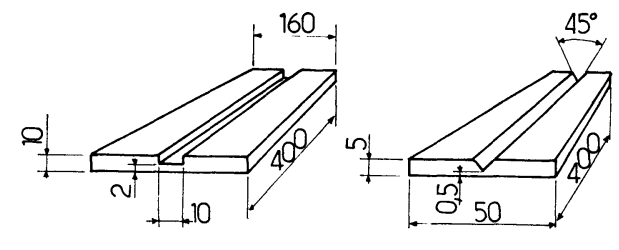

Type I

Type $\mathbb{I}$

FIg. 4. - Les deux types de plaques utilisées (cotes en $\mathrm{mm}$ ).

résistance totale du circuit tout en présentant localement une section droite de $8 \mathrm{~cm}^{2}$. La largeur de la rainure $(1 \mathrm{~cm})$ a été choisie pour augmenter la tension d'arc. Toutefois la découpe réalisée n'autorise pas une évacuation rapide des calories de la zone centrale vers le reste de la plaque.

Afin de permettre la diffusion thermique la plaque est rainurée en $\mathrm{V}$, modification qui conduit à l'emploi de pièces plus minces et plus étroites (Fig. 4, type II), tout en conservant, à peu près, la même résistance électrique aux bornes de la dynamo homopolaire. Après quelques essais nous constatons que la section droite nécessaire au fonctionnement de l'interrupteur n'est plus que de $2 \mathrm{~cm}^{2}$.

3. Résultats. - 3.1 SURTENTION ET TEMPS DE COUPURE. - La dispersion des résultats étant relativement faible, environ $10 \%$ sur les grandeurs mesurées, nous donnons sur la figure 5 les courbes typiques de tension

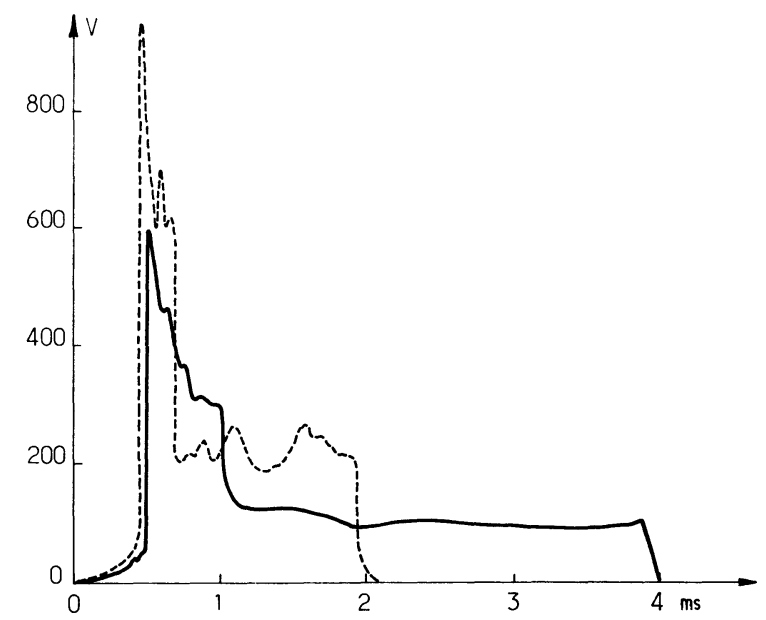

Fig. 5. - Courbes caractéristiques des tensions obtenues aux bornes de l'interrupteur (extrait de cliché polaroïd). — type I ; - . - type II.

obtenues avec les plaques du type I et du type II. Dans les deux cas, la coupure du courant de 0,5 MA a effectivement lieu, à quelques millisecondes près, au moment du maximum de courant. Ce décalage en temps est négligeable devant le temps de montée de l'impulsion de courant $(\sim 1 \mathrm{~s})$ et le courant varie très peu au voisinage de son maximum. 
Dans l'ensemble les deux courbes ont le même aspect : au moment de la rupture du fusible la tension monte rapidement (quelques dizaines de microsecondes) décroît peu pendant un temps inférieur à la milliseconde, puis l'arc s'établit avec chute de la tension qui demeure constante jusqu'à extinction de l'arc.

Lors de l'utilisation de plaques du type I, la tension crête atteint $600 \mathrm{~V}$ et le temps total d'ouverture est de $4 \mathrm{~ms}$.

Avec des plaques du type II, la tension crête atteint $960 \mathrm{~V}$ et le temps total d'ouverture est de $2 \mathrm{~ms}$.

3.2 Processus D'ouverture. - L'observation des plaques rompues nous renseigne sur le processus de la coupure.

Nous savons que les densités de courant sont plus grandes aux extrémités de la bobine qu'au centre [2]. La théorie donne un facteur voisin de 2. La plaque fond d'abord aux extrémités, le long d'une génératrice, comme nous l'observons dans le cas où il n'y a pas rupture par insuffisance du courant total en raison d'une résistance d'interrupteur un peu supérieur à la moyenne.

Les parties fondues présentent une résistance très élevée, les courants locaux sont alors déviés vers le centre de la plaque. Ils sont parallèles et en sens contraire, les forces de Laplace $\left(\mathbf{F}_{2}, \mathbf{F}_{3}\right)$ tendent à ouvrir le sillon formé par la fusion et la force $\mathbf{F}_{1}$, due à l'induction magnétique, aide à l'allongement de ce sillon (Fig. 6).

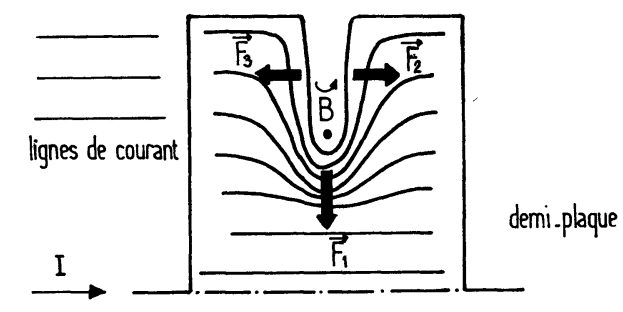

Fig. 6. - Processus de découpage des plaques.

Le processus se termine par l'explosion de la partie centrale où la densité de courant est devenue trop importante $10^{7} \mathrm{~A} . \mathrm{cm}^{-2}$ ), comme l'aspect des lèvres de la coupure en témoignent : lisses aux extrémités, arrachées au centre.

Ce phénomène d'écartement n'est pas entravé dans le montage utilisant les plaques du type II et après expérience les morceaux de la plaque sont distants de $4 \mathrm{~cm}$ à leurs extrémités et cintrés (photo) en raison des vis de montage. Le soufflage de l'arc, par l'induction magnétique régnant dans la bobine, est complété par l'allongement de l'arc dû à l'écartement des lèvres de la coupure. Cela explique une tension au moment du plateau plus élevée pour le type II que pour le type I, donc à énergie stockée égale un temps d'ouverture plus court (Fig. 5).

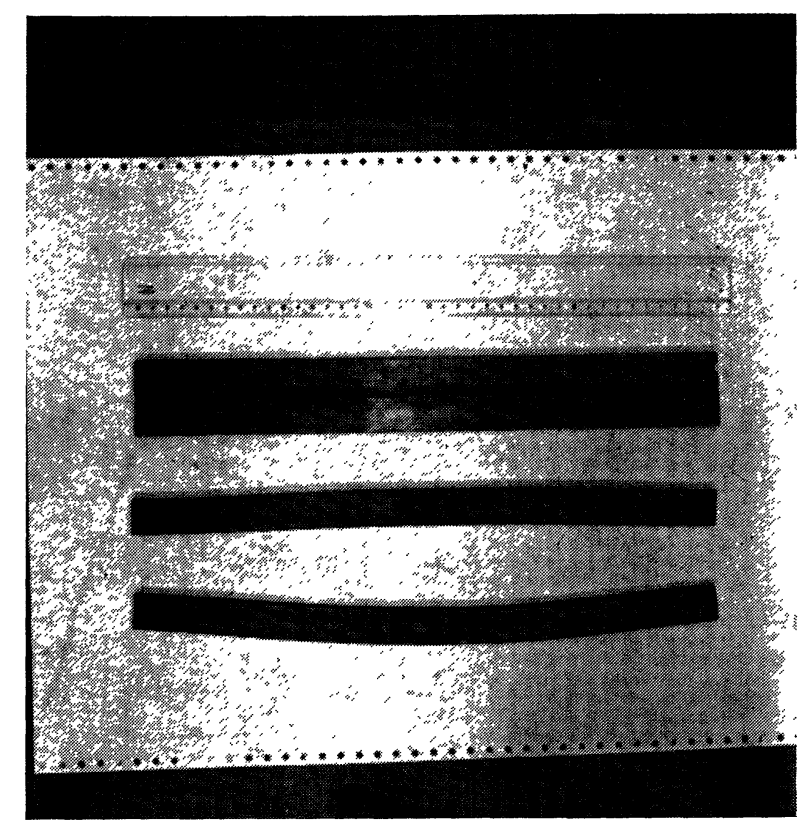

Pното. - Une plaque du type II avant et après rupture.

Un calcul approché du travail des forces $\mathbf{F}_{2}$ et $\mathbf{F}_{\mathbf{3}}$ donne un temps d'écartement pour $4 \mathrm{~cm}$ de l'ordre de la milliseconde et dans l'expérience la durée totale d'ouverture est de $1,5 \mathrm{~ms}$.

La différence qui existe entre les deux types de plaques, en ce qui concerne la première partie de la courbe de tension, est liée en particulier au phénomène d'écartement et à la quantité de cuivre consommé qui est de l'ordre de $0,2 \mathrm{~cm}^{3}$ pour le type II mais atteint $8 \mathrm{~cm}^{3}$ pour le type I (toute la partie amincie).

4. Etude énergétique. - La coupure du courant s'effectue, ainsi que nous venons de le voir, en deux phases pendant lesquelles, l'énergie stockée dans la bobine (de l'ordre de $100 \mathrm{~kJ}$ ) est dissipée ; examinons dans quelles proportions.

Nous mesurons la dérivée du courant, $\mathrm{d} I / \mathrm{d} t$, et la tension $U$ aux bornes de l'interrupteur. Ces deux grandeurs sont proportionnelles $\left(U=-L \frac{\mathrm{d} I}{\mathrm{~d} t}\right.$ où $L$ est l'inductance de la bobine). Par intégration nous obtenons la variation du courant après rupture du circuit. De la mesure de $U$ et du calcul de $I$ nous déduisons la variation de la puissance mise en jeu à chaque instant, les différentes courbes sont présentées, pour une plaque du type II sur la figure 7.

Le courant chute rapidement de $420 \mathrm{kA}$ à $260 \mathrm{kA}$ en $260 \mu$ s puis décroît lentement. De ces deux phases, la première est la plus importante, car c'est au moment de la rupture du fusible que le transfert d'énergie magnétique aura lieu. Au cours de cette phase, près des deux tiers de l'énergie stockée sont absorbés, le reste est dissipé dans l'arc au cours de la deuxième phase. Il faut noter que pour les plaques du type I nous obtenons les mêmes proportions. 


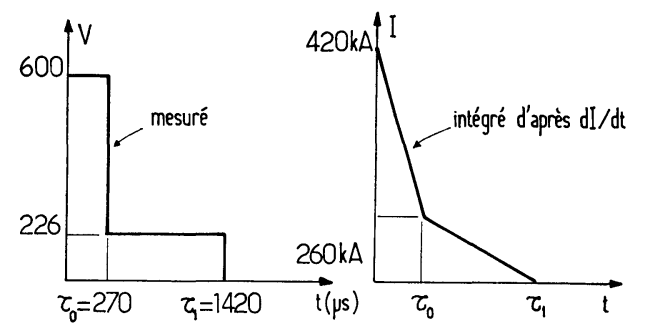

(a)

(b)

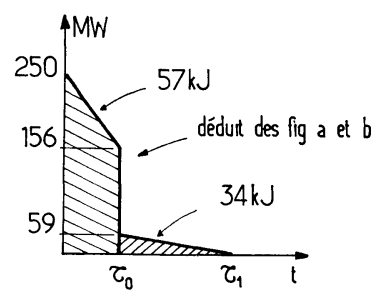

(c)

Fig. 7. - Schémas des tension, courant et puissance.

L'énergie nécessaire pour porter le cuivre de l'état solide, à température ambiante, à l'état liquide est de 10 à $12 \mathrm{~kJ} . \mathrm{cm}^{-3}$, pour atteindre l'état vapeur il faut $50 \mathrm{~kJ} . \mathrm{cm}^{-3}$.

Dans le cas de plaques du type $\mathrm{I}$, où la perte de matière est de $8 \mathrm{~cm}^{3}$, le processus de rupture ne passe pas par la vaporisation du cuivre, ce qui nécessiterait $400 \mathrm{~kJ}$; ceci est toutefois moins évident pour les fusibles du type II qui consomment environ $0,2 \mathrm{~cm}^{3} \mathrm{de}$ cuivre soit $10 \mathrm{~kJ}$. Les phénomènes mis en jeu ne sont donc pas comparables à ceux rencontrés dans le cas de feuilles explosées [3].

5. Comparaison avec les feuilles explosées. - Les feuilles explosées sont utilisées pour ouvrir des circuits dans lesquels la source de courant est un générateur à compression de champ magnétique, à explosif solide, alimenté par la décharge de condensateur [3].

L'impulsion de courant atteint le mégaampère en quelques dizaines de microsecondes. L'ouverture du circuit par explosion de feuilles de cuivre minces $(25 \mu \mathrm{m}$ à $100 \mu \mathrm{m}$ ) s'effectue en un temps très court (quelques microsecondes). Les meilleurs résultats donnent des coefficients de surtension de 10 , le plus souvent 3 à 4 en prenant pour référence la tension de charge des condensateurs.

Dans le cas d'une génératrice homopolaire, le temps de montée de l'impulsion de courant, voisin de la seconde, ne permet pas l'utilisation de feuilles minces. La tension aux bornes du fusible avant ouverture du circuit est inférieur au volt : le coefficient de surtension est donc supérieur à 500 .

La feuille de cuivre est entièrement vaporisée alors que seule une partie du fusible l'est.

Nous pensons que les deux procédés sont complémentaires dans le cas d'une source délivrant son énergie en un temps long (dynamo impulsionnelle). Le fusible servant d'interrupteur primaire (Fig. 1), un dispositif à feuilles explosées peut être monté aux bornes de la bobine $\mathrm{B}_{2}[4,5,6]$.

6. Etude théorique. - Pour améliorer les résultats obtenus, il faut connaître les phénomènes entrant en jeu lors de la rupture du fusible, en particulier, l'évolution de la température en différents points en fonction de divers paramètres : épaisseur, angle d'ouverture de la rainure...

Nous avons donc entrepris un calcul plus élaboré que celui qui nous a guidé lors des premiers essais (type I) où l'équation utilisée était

$$
R I^{2}(t) \mathrm{d} t=M C \mathrm{~d} \theta
$$

$R$ est la résistance du fusible, $M$ sa masse, $C$ la chaleur massique du cuivre solide et $\theta$ la température, $I(t)$ le courant total; relation qui devient :

$$
\rho(\theta) \frac{I^{2}(t)}{s^{2}} \mathrm{~d} t=\bar{\omega} C \mathrm{~d} \theta
$$

où $\rho(\theta)$ est la résistivité du cuivre, $s$ la section droite du fusible et $\bar{\omega}$ la masse volumique du cuivre.

En tenant compte de la diffusion thermique la relation ci-dessus devient :

$$
\begin{aligned}
& \rho(\theta) \frac{I^{2}(t)}{s(x)}= \\
& =s(x) \bar{\omega} C \frac{\mathrm{d} \theta}{\mathrm{d} t}-\lambda \frac{\partial}{\partial x}\left(\frac{\partial \theta}{\mathrm{d} x} s(x)\right)+\frac{2 h\left[s(x)+L^{2}\right]}{L}\left(\theta-\theta_{0}\right)
\end{aligned}
$$

où $s(x)$ est la section du fusible variable avec $x$ (l'axe des $x$ est parallèle au courant) $\lambda$ le coefficient de diffusion thermique dans le cuivre, $h$ le coefficient d'échange thermique par convection cuivre-air et $L$ la hauteur de la plaque $(400 \mathrm{~mm})$.

L'introduction de l'inhomogénéité des courants locaux, ceux-ci sont fonction de $y$, rend le calcul relativement complexe ; cependant, conduit jusqu'à obtention de la température de fusion au bord de la rainure ce calcul a déjà donné des résultats intéressants qui sont présentés sur les figures 8 à 10 pour les plaques du type II.

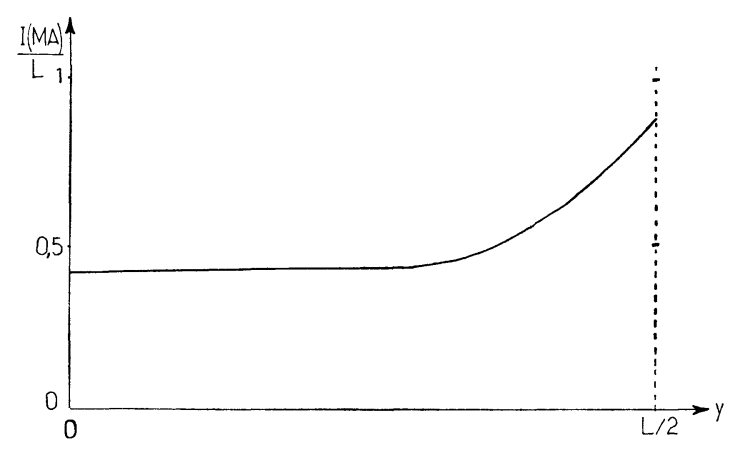

FIg. 8. - Répartition des courants le long de la rainure à l'instant du maximum du courant total. 


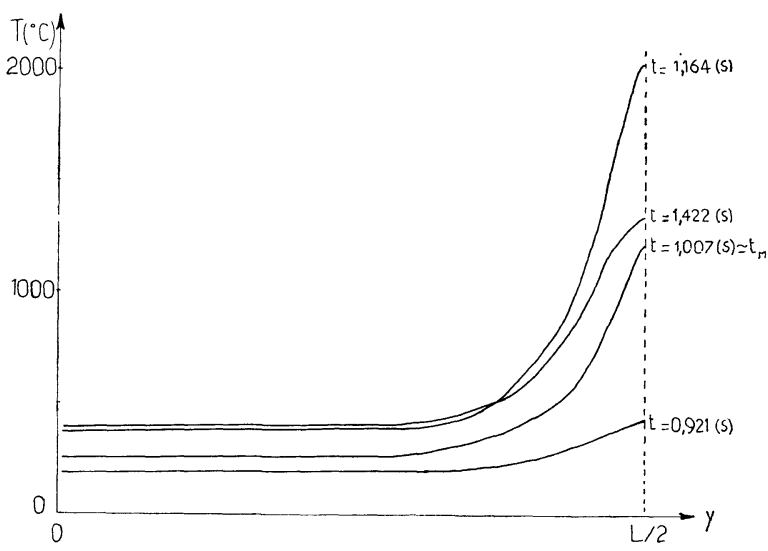

Fig. 9. - Evolution de la température en fonction du temps le long de la rainure.

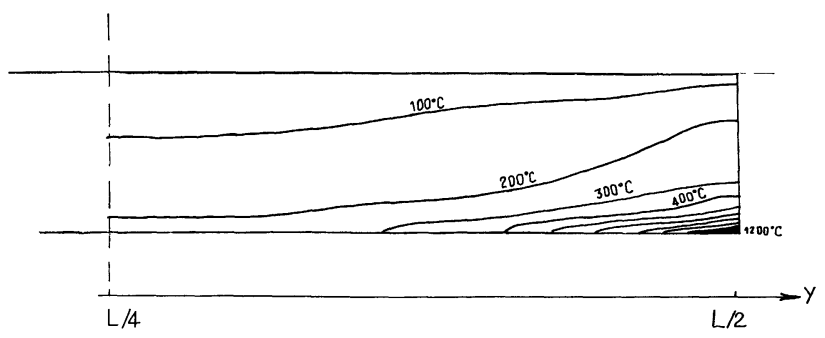

Fig. 10. - Isothermes lorsque l'extrémité du fusible atteint la température de fusion.

La figure 8 montre la répartition des courants locaux à l'instant du maximum du courant total, le long de la rainure (demi-plaque) s'il n'y avait pas rupture. Le courant total atteint 0,5 MA.
La figure 9 donne l'évolution de la température le long de la rainure pour différents temps ( $t_{\mathrm{M}}$ est l'instant du maximum du courant total).

Sur la figure 10 sont tracées les isothermes à l'instant où le bord de la rainure atteint le point de fusion.

L'accord est parfait avec les explications qualitatives de la progression de la rupture.

Nous envisageons la détermination des formes optimales des plaques à utiliser (rendement énergétique maximum, temps de rupture minimum). Le rendement énergétique maximum exige une quantité de cuivre vaporisée minimum et le temps nous impose une concentration accélérée des lignes de courant, ce qui sera peut être obtenu en effectuant une pré-découpe des plaques le long de la rainure.

Ces calculs seront publiés ultérieurement $\left({ }^{1}\right)$.

7. Conclusion. - Nous avons expérimenté deux sortes d'interrupteurs du type fusible et nous avons effectivement coupé le courant de 0,5 MA dans des temps de l'ordre de la milliseconde.

Nous avons également montré que le processus de coupure n'est pas celui rencontré avec des feuilles explosées. Nous avons ici un coefficient de surtension qui dépasse 500 alors qu'avec les feuilles ce coefficient est toujours inférieur à 10 .

Dans le montage final avec couplage inductif des bobines $\mathbf{B}_{1}$ et $\mathbf{B}_{2}$ l'énergie à dissiper dans l'interrupteur sera bien plus petite que celle que nous avons dissipée dans nos essais où toute l'énergie magnétique stockée dans la bobine doit être absorbée dans l'arc. Aussi, dans le transfert inductif seul le début de la courbe de tension sera utilisé.

Les calculs en cours nous permettrons, probablement, d'améliorer les résultats déjà obtenus.

\section{Bibliographie}

[1] Hahn, R., Guillet, R., Lucidarme, J., Rioux, C., Revue Phys. Appl. 8 (1973) 53.

[2] Geoffrion, B., Legentil, M., Revue Phys. Appl. 10 (1975) 11.

[3] Antoni, B., Revue Phys. Appl. 9 (1974) 331.
[4] LegentIL, M., Rapport Interne LF 18 (1973).

[5] Rioux Damidau, F., Revue Phys. Appl. 10 (1975) 27.

[6] Rioux, C., Revue Phys. Appl. 10 (1975) 75. 